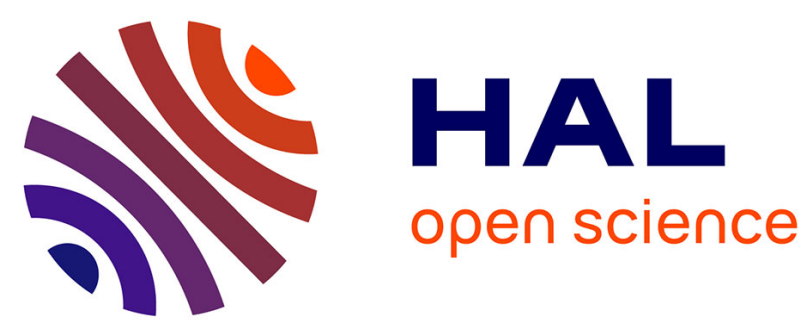

\title{
Normal forms of matrix words for stability analysis of discrete-time switched linear systems
}

Cyrille Chenavier, Rosane Ushirobira, Laurentiu Hetel

\section{To cite this version:}

Cyrille Chenavier, Rosane Ushirobira, Laurentiu Hetel. Normal forms of matrix words for stability analysis of discrete-time switched linear systems. ECC 2020 - 18th European Control Conference, May 2020, Saint Petersburg, Russia. 10.23919/ECC51009.2020.9143862 . hal-02069712v3

\section{HAL Id: hal-02069712 \\ https://hal.science/hal-02069712v3}

Submitted on 18 Feb 2020

HAL is a multi-disciplinary open access archive for the deposit and dissemination of scientific research documents, whether they are published or not. The documents may come from teaching and research institutions in France or abroad, or from public or private research centers.
L'archive ouverte pluridisciplinaire HAL, est destinée au dépôt et à la diffusion de documents scientifiques de niveau recherche, publiés ou non, émanant des établissements d'enseignement et de recherche français ou étrangers, des laboratoires publics ou privés. 


\title{
Normal forms of matrix words for stability analysis of discrete-time switched linear systems
}

\author{
Cyrille Chenavier, Rosane Ushirobira, Laurentiu Hetel
}

\begin{abstract}
In this paper, we propose a new method for investigating the stability of discrete-time switched linear systems by means of linear algebra techniques. Exponential stability of such systems is equivalent to the existence of solutions of linear matrix inequalities indexed by matrix words (i.e. products of matrices of the sub-systems). Our method consists in using the link between this characterization of exponential stability and linear dependency among matrix words. In particular, we introduce a criterion to reduce drastically the number of linear matrix inequalities, by removing redundant ones. This is achieved by eliminating matrix words that depend on the others. From this criterion, we also relate exponential stability to quadratic stability of another switched system. An example is given to illustrate our methods.
\end{abstract}

\section{INTRODUCTION}

In this article, we investigate some properties of discretetime switched linear systems [1], [2]. Such systems consist of a family of linear systems with a rule that orchestrates the switching among them. Discrete-time switched linear systems are a popular model in various control domains. Among the application areas, we may cite networked control systems [3], systems with aperiodic sampling [4], discretetime delay systems [5], [6], etc., they all can be modeled as switched linear systems. Despite the fact that switched systems have been intensively studied over the last two decades, the stability problem is still a complex open problem [7].

Various methods are available for studying the stability of discrete-time switched linear systems. Roughly speaking, there are stability criteria that have been proposed based on algebraic methods, such as the ones within the framework of the theory of Lie algebras [8], [9] or the ones based on joint spectral radii [10], [11]. Numerical approaches, usually based on Linear Matrix Inequalities (LMI) and Lyapunov functions were also given in different works. In this direction, stability criteria have been proposed by checking the existence of quasi-quadratic [12], [13], parameter dependent [14], pathdependent [15], non-monotonic [16], [17], [18], [19], [20], with an augmented state vector [21], composite quadratic [13], [22], [23] Lyapunov functions or using a Gaussian elimination procedure [24]. The aim of the present article is

The first two authors are with Inria, Univ. Lille, CNRS, UMR 9189 CRIStAL - Centre de Recherche en Informatique Signal et Automatique de Lille, F-59000 Lille, France. Laurentiu Hetel is with CNRS, Univ. Lille, UMR 9189 - CRIStAL - Centre de Recherche en Informatique Signal et Automatique de Lille, F-59000 Lille, France. to propose a new method to analyze the stability of switched linear systems by investigating the structural and algebraic properties of some particular Lyapunov based criteria.

A remarkable result, that can be found in [17], provides a necessary and sufficient condition for a discrete-time switched linear system to be globally uniformly exponentially stable: it is equivalent to the existence of a positive integer $N$ such that a particular problem consisting in $p^{N}+1$ LMI conditions admits a solution. From this condition, it follows the construction of the quasi-quadratic Lyapunov function: starting with $N=1$, the value of $N$ is increased until a solution to the LMI problem can be found. From that, the corresponding quasi-quadratic Lyapunov function is derived. As mentioned in [22], a significant improvement of this method would be to reduce the computational complexity, since the number of LMI conditions grows exponentially when $N$ increases.

In this work, starting with the condition proposed in [17], we use tools from linear algebra to introduce two sufficient conditions for proving stability of study discretetime switched linear systems. Two criteria are provided. These two criteria are based on the remark that the matrices involved in the LMI problem from [17] may satisfy algebraic relations. So our first method consists in restricting the LMI conditions to a smaller problem, by reducing it to a basis of the linear span of all matrices involved in the initial LMI problem. Next, we must check if the LMI solution for the basis elements still holds for the remaining original matrices. The second method is also based on the basis: it consists in adding numerical constraints to the LMIs corresponding to basis elements. Thanks to this criterion, the remaining LMI conditions corresponding to the other matrices are automatically fulfilled. The main argument used in this second approach is that the convex decomposition of matrices does not bring new LMI constraints. Moreover, it should be stressed that for both proposed methods, the number of LMI conditions is upper-bounded by the dimension of the matrix space, which does not depend on $N$.

The paper is organized as follows. In Section II, some useful notions are given. In Section III, we recall notions about discrete-time switched linear systems, stability definition and its characterization in terms of LMIs given in [17]. Section IV contains our results: we present two 
novel methods for reducing the number of LMI conditions, the first one using linear algebra and the second one using convex decomposition. In Section V, the second method is illustrated with a numerical example. Section VI contains the proof of an intermediate lemma.

\section{PRELIMINARIES}

Definition 2.1: Given an arbitrary set $S$, a word $w$ over $S$ is a finite formal concatenation of elements of $S$ :

$$
\omega=s_{1} s_{2} s_{3} \ldots s_{N}
$$

where $s_{j} \in S$, for all $j \in\{1, \cdots, N\}$.

The set $S$ is called an alphabet. We denote by $S^{*}$ the set of words over $S$ and by $S^{(N)}$ the set of words of $S^{*}$ of length $N$ for a given $N \in \mathbb{N}$, so that $S^{(0)}$ is reduced to the empty word and $S^{(1)}=S$.

Example 2.2: As an illustration, consider $S=\{a, b\}$. Then

$$
\begin{aligned}
S^{(2)} & =\{a a, a b, b a, b b\} \\
S^{(3)} & =\{a a a, a a b, a b a, a b b, b a a, b a b, b b a, b b b\}
\end{aligned}
$$

The notion of words can be naturally applied to the set of matrices, as in the following definition.

Definition 2.3: Let $p, n \in \mathbb{N} \backslash\{0\}$. Set $\mathcal{J}_{p}:=\{1, \cdots, p\}$ and consider a set of matrices $\mathcal{A}=\left\{A_{i} \in \mathbb{R}^{n \times n} \mid i \in \mathcal{J}_{p}\right\}$. Given a word $\omega=i_{k-1} \cdots i_{0} \in \mathcal{J}_{p}^{*}$ on the alphabet $\mathcal{J}_{p}$, the corresponding matrix word on $\mathcal{A}$ is denoted by

$$
A_{\omega}=A_{i_{k-1}} \cdots A_{i_{0}} \in \mathcal{A}^{*}
$$

where the concatenation of matrices stands for the matrix product. By convention, if $k=0$, we let $A_{\omega}=\operatorname{Id}_{n}$ where $\operatorname{Id}_{n}$ denotes the identity matrix of order $n$.

Example 2.4: If $p=2$, then $\mathfrak{J}_{2}=\{1,2\}$. Consider $\mathcal{A}=$ $\left\{A_{1}, A_{2}\right\}$ with

$$
A_{1}=\left(\begin{array}{ll}
1 & 1 \\
0 & 1
\end{array}\right), \quad A_{2}=\left(\begin{array}{ll}
0 & 1 \\
1 & 0
\end{array}\right)
$$

The set of words of length 2 in $J_{2}$ is $J_{2}^{(2)}=\{11,12,21,22\}$. So there are four matrix words of length 2 corresponding to the words in $\mathcal{J}_{2}^{(2)}$, they are:

$$
\begin{aligned}
& A_{11}=A_{1} A_{1}=\left(\begin{array}{ll}
1 & 2 \\
0 & 1
\end{array}\right), \\
& A_{12}=A_{1} A_{2}=\left(\begin{array}{ll}
1 & 1 \\
1 & 0
\end{array}\right), \\
& A_{21}=A_{2} A_{1}=\left(\begin{array}{ll}
0 & 1 \\
1 & 1
\end{array}\right), \\
& A_{22}=A_{2} A_{2}=\mathrm{Id}_{2} .
\end{aligned}
$$

\section{Problem Statement}

In this Section, a stability problem for a class of discretetime switched linear systems is presented. Let $n$ and $p$ be two strictly positive integers and a set $\mathcal{A}$ of $p$ matrices $A_{1}$, $A_{2}, \ldots, A_{p}$. Consider the following switched linear system:

$$
x_{k+1}=A_{\sigma_{k}} x_{k}, \forall k \in \mathbb{N}, x_{0} \in \mathbb{R}^{n},
$$

where $x: \mathbb{N} \rightarrow \mathbb{R}^{n}$ represents the system state, $x_{0}=x(0)$ the initial condition and $\sigma: \mathbb{N} \rightarrow \mathcal{J}_{p}, k \mapsto \sigma_{k}$ the switching function associated to the system. It is assumed that the switching function is not known.

For $k \in \mathbb{N}$, denote by $\mathcal{S}^{(k)}=\mathcal{S}^{(k)}\left(\mathcal{J}_{p}\right) \subset \sigma(\mathbb{N})^{*}$ the set of $k$ length words formed by the image of the switching function $\sigma$ starting with $\sigma_{0}$. So, an element of $\mathcal{S}^{(k)}$ is a word of the form $\sigma_{i_{k-1}} \ldots \sigma_{i_{1}} \sigma_{0}$ with $\sigma_{i_{j}}=\sigma\left(i_{j}\right), i_{j} \in \mathcal{J}_{p}$. For example, with the notation in Example 2.4 and a switching law $\sigma$ defined by $\sigma(i)=1$ if $i$ is odd and 2 if $i$ is even, for all $i \in \mathbb{N} \backslash\{0\}, \sigma_{0}=1$, the elements of $\mathcal{S}^{(2)}$ are 11,21 . This set is also called the set of $k$-length switching paths.

For the initial condition $x_{0} \in \mathbb{R}^{n}$ and an infinite word $\omega=\left(\sigma_{k}\right)_{k=0}^{\infty} \in \mathcal{S}^{\infty}$, we consider the flow $\left(k, x_{0}\right) \mapsto \phi_{\omega}\left(k, x_{0}\right)$ defined by

$$
\phi_{\omega}\left(k, x_{0}\right)=A_{\omega}(k) x_{0},
$$

where $A_{\omega}(k)=A_{\sigma_{k-1}} \cdots A_{\sigma_{0}} \in \mathbf{A}^{*}=\mathcal{A}^{*}$, so that $A_{\omega}(0)=\operatorname{Id}_{n}$, is called the $k$-step transition matrix of the discrete-time switched system associated to $\omega$.

For an infinite word $\omega \in \mathcal{S}^{\infty}$, the solution of (1) associated to the initial condition $x_{0}$ is given by the sequence of points $\left(\phi_{\omega}\left(k, x_{0}\right)\right)_{k=0}^{\infty}$.

Next, some notations are set and classical definition recalled. So, given a matrix $M \in \mathbb{R}^{n \times p}, M^{\top} \in \mathbb{R}^{p \times n}$ denotes the transpose of $M$. For $x=\left(x_{1} \ldots x_{n}\right)^{\top} \in \mathbb{R}^{n}$, we denote by $\|x\|=\sqrt{x_{1}^{2}+\cdots+x_{n}^{2}}$ the Euclidean vector norm on $\mathbb{R}^{n}$.

Definition 3.1: The equilibrium point $x=0$ of the switched linear system (1) is globally uniformly exponentially stable (GUES) if there exists a constant $c>0$ and $0<\lambda<1$ such that

$$
\left\|\phi_{\omega}\left(\ell, x_{0}\right)\right\|^{2} \leq c \lambda^{\ell}\left\|x_{0}\right\|^{2}
$$

holds for all initial conditions $x_{0} \in \mathbb{R}^{n}$, all $\ell \in \mathbb{N}$ and all sequences of switching $\omega=\left(\sigma_{k}\right)_{k=0}^{\infty} \in \mathcal{S}^{\infty}$.

The following well-known stability criteria can be found for instance in [17]. Note that given a square symmetric matrix $M$, we write $M \succ 0$ or $M \prec 0$ accordingly if $M$ is positive or negative definite.

Proposition 3.2: The following assertions are equivalent:

1) The equilibrium point $x=0$ of (1) is GUES

2) There exists a positive integer $N$ and a matrix $P=$ $P^{T} \succ 0$ such that

$$
P \succ A_{\omega}^{T} P A_{\omega}, \forall \omega \in \mathcal{S}^{(N)} .
$$


As an immediate corollary, we obtain the following criterion for checking the GUES property:

Corollary 3.3: Let $N$ be a positive integer. If the LMI problem (3) admits a solution, then the equilibrium point $x=0$ of (1) is GUES.

It is a straightforward conclusion that for a fixed $N$, the LMI criterion of Proposition 3.3 for checking the GUES property consists of $p^{N}+1$ LMI conditions. In Section IV, a method to simplify Proposition 3.3 is proposed. This new approach is based on linear algebra concepts and it aims to drastically reduce the number of LMI conditions.

\section{CONDITIONS BASED ON NORMAL FORM OF MATRIX WORDS}

In this section, a new algebraic method for analyzing the GUES property is presented. The main idea is to take into consideration linear dependency between matrix words, that is the elements of $\mathcal{A}^{*}$. As an illustration of this approach, we show in Theorem 4.5 that the number of LMI conditions in (3) may be reduced and become independent from the length of matrix words.

For that, matrix words $A_{\omega} \in \mathcal{A}^{*}$ are realized as elements of the vector space $\mathbb{R}^{n \times n}$. Since any vector space has a basis, it may exist a matrix word $A_{\omega_{0}} \in \mathcal{A}^{*}$ which is a linear combination of other matrix words of the same length, that is

$$
A_{\omega_{0}}=\sum \mu_{\omega} A_{\omega}
$$

for scalars $\mu_{\omega} \in \mathbb{R}$. So, our method consists in rewriting the conditions (3) without taking into account the redundant inequalities corresponding to the words such as in (4). Moreover, we wish to consider only LMIs associated to matrix words $A_{\omega}$ that form a basis of the subspace of $\mathbb{R}^{n \times n}$ generated by all matrix words of the same length in $\mathcal{A}^{*}$ (those for which no equality of the form (4) holds). This is possible when the parameters $\mu_{\omega}$ in (4) satisfy particular numerical constraints as we shall see in Theorem 4.5.

Given $N$, let us denote by $V_{\mathcal{A}}^{(N)}$ the subspace of $\mathbb{R}^{n \times n}$ spanned by the matrices $A_{\omega}$, where $\omega \in \mathcal{J}_{p}^{(N)}$.

Definition 4.1: Let $d_{N}$ be the dimension of $V_{\mathcal{A}}^{(N)}$ and let $A_{\omega_{1}}, \cdots, A_{\omega_{d_{N}}} \in \mathcal{A}^{*}$, be a basis of $V_{\mathcal{A}}^{(N)}$ composed of matrix words, that is $\omega_{i} \in \mathcal{J}_{p}^{(N)}$. The matrices $A_{\omega_{i}}\left(1 \leq i \leq d_{N}\right)$ will be called normal form matrices.

Next, non-normal form matrices are denoted by $A_{\omega_{i}}$, for all $d_{N}+1 \leq i \leq p^{N}$.

Since the vector space spanned by normal form matrices is a subspace of the $n^{2}$-dimensional space $\mathbb{R}^{n \times n}$, the following lemma holds immediately:

Lemma 4.2: The number $d_{N}$ of normal form matrices is smaller than $n^{2}$.

Remark 4.3: Given a positive integer $N$, a necessary condition for the existence of a matrix $P$ solution of the LMIs problem (3) is that the following LMI problem admits a solution:

there exists $P=P^{T} \succ 0$, such that $P \succ A_{\omega_{i}}^{T} P A_{\omega_{i}}, \forall 1 \leq i \leq d_{N}$.

Remark 4.3 has two important consequences. On the one hand, if (5) has no solution, then the criterion of Proposition 3.3 proving the GUES property does not hold. On the other hand, if (5) admits a matrix $P$ as a solution such that $P \succ A_{\omega_{i}}^{T} P A_{\omega_{i}}$ for each non-normal form matrix $A_{\omega_{i}}$, then the criterion of Proposition 3.3 holds.

Moreover, from Lemma 4.2, the number of normal form matrices is upper-bounded by $n^{2}$, so that the maximal number of LMI conditions of (5) does not depend on $N$. However, a solution of (5) is not necessarily a solution of (3). Our objective is to introduce a LMI problem with the same number of conditions than (5) and for which any solution is automatically a solution of (3).

We fix a positive integer $N$, and we denote as in Definition 4.1, normal form matrices and non-normal form matrices by $A_{\omega_{1}}, \cdots, A_{\omega_{d_{N}}}$ and $A_{\omega_{d_{N}+1}}, \cdots, A_{\omega_{p^{N}}}$, respectively. For each non-normal form matrix $A_{\omega_{\ell}}$, let $\lambda_{\ell, 1}, \cdots, \lambda_{\ell, d_{N}}$ be its coordinates in the basis of normal form matrices, so that we have

$$
A_{\omega_{\ell}}=\sum_{k=1}^{d_{N}} \lambda_{\ell, k} A_{\omega_{k}}
$$

For $1 \leq i \leq d_{N}$, we define the real number:

$$
m_{i}=\max _{1 \leq \ell \leq p^{N}}\left\{\sum_{k=1}^{d_{N}}\left|\lambda_{\ell, k}\right| \mid \lambda_{\ell, i} \neq 0\right\}
$$

Notice that $m_{i} \geq \lambda_{\ell, i}$ for all $i, \ell$.

Before stating our main result, we need the following Lemma, for which a proof is given the Appendix:

Lemma 4.4: Let $P=P^{T} \succ 0$ be such that $P \succ m_{i} A_{\omega_{i}}^{T} P A_{\omega_{i}}$, for every $1 \leq i \leq d_{N}$. Then, we have

$$
P \succ A_{\omega_{\ell}}^{T} P A_{\omega_{\ell}}, \forall 1 \leq \ell \leq p^{N} .
$$

We obtain our main result, stated as follows:

Theorem 4.5: Consider the system (1). Let $N$ be a positive integer and $A_{\omega_{1}}, \cdots, A_{\omega_{d_{N}}}$ be the normal form matrices. For $d_{N}+1 \leq \ell \leq p^{N}$, let $\lambda_{\ell, k}\left(1 \leq k \leq d_{N}\right)$ be the coefficients of the decomposition of the non-normal form matrix $A_{\omega_{\ell}}$ (see (6)). Consider the real number defined in (7):

$$
m_{i}=\max _{1 \leq \ell \leq p^{N}}\left\{\sum_{k=1}^{d_{N}}\left|\lambda_{\ell, k}\right| \mid \lambda_{\ell, i} \neq 0\right\}, \forall 1 \leq i \leq d_{N} .
$$

If the LMI problem

$$
\exists P=P^{T} \succ 0, P \succ m_{i} A_{\omega_{i}}^{T} P A_{\omega_{i}}, 1 \leq i \leq d_{N},
$$

admits a solution, then the equilibrium point $x=0$ of (1) is GUES. 
Proof: Assume that (9) admits a solution. From (8), we have $P \succ A_{\omega_{\ell}}^{T} P A_{\omega_{\ell}}$ for every $1 \leq i \leq p^{N}$. Hence, $P$ is a solution of (3), so that (1) is GUES from Proposition 3.3.

Remark 4.6: We fix an integer $N$.

1) The existence of a solution for the LMI problem (9) does not mean that the system (1) is quadratically stable, excepted if $N=1$. A counter-example is given in Section V.

2) Theorem 4.5 shows that the stability of (1) is related to the quadratic stability of another discrete-time linear switched system, namely

$$
z_{k+1}=A_{\omega_{\gamma_{k}}} z_{k}, \forall k \in \mathbb{N}, z_{0} \in \mathbb{R}^{n},
$$

with the switching function $\gamma: \mathbb{N} \rightarrow \mathcal{J}_{d_{N}}$. More precisely, when (9) admits a solution, (10) is quadratically stable and an estimation of the decay rate equals to $\sqrt{\delta}$, where

$$
\delta=\max _{1 \leq i \leq d_{N}}\left\{\frac{1}{m_{i}}\right\} .
$$

\section{EXAMPLES}

In this section, we illustrate Theorem 4.5 with an example adapted from [25], [22]: we consider two matrices

$$
A_{1}^{c}=\left(\begin{array}{cc}
-1 & -1 \\
1 & -1
\end{array}\right) \text { and } A_{2}^{c}=\left(\begin{array}{cc}
-1 & -a \\
\frac{1}{a} & -1
\end{array}\right)
$$

where $a \in \mathbb{R}_{>0}$ and we consider the discrete-time switched linear system (1) with $p=2$ and $A_{i}=\exp \left(A_{i}^{c} T\right)$ with $T=1$ for $i \in J_{2}=\{1,2\}$. So $\mathbf{A}=\left\{A_{1}, A_{2}\right\}$.

The following table was given in [22, Section V]:

\begin{tabular}{|c|c|c|c|c|c|}
\hline & $\mathrm{a}=5$ & $\mathrm{a}=6$ & $\mathrm{a}=7$ & $\mathrm{a}=8$ & \#LMI conditions \\
\hline $\mathrm{N}=1$ & $\checkmark$ & - & - & - & 2 \\
\hline $\mathrm{N}=3$ & $\checkmark$ & $\checkmark$ & - & - & 9 \\
\hline $\mathrm{N}=8$ & $\checkmark$ & $\checkmark$ & $\checkmark$ & $\checkmark$ & 257 \\
\hline
\end{tabular}

The meaning is the following: for every box with a $\checkmark$, a matrix $P$ for the corresponding LMI problem (3) was obtained and for every box with - , no matrix was obtained ${ }^{1}$.

Moreover, each value of $N$ corresponds to the smallest one for which a matrix $P$ was obtained for a new value of $a$ : in particular, for $a=7,8$ and $4 \leq N \leq 7$, no matrix were obtained.

The method presented in the previous section consists in extracting a basis of the linear span of $A_{\omega}$, for a fixed length of words. Given values $5 \leq a \leq 8$ and $2 \leq N \leq 8$, the matrices $A_{\omega 11}, A_{\omega 12}, A_{\omega 21}, A_{\omega 22}$, where the length of $\omega$ is $N-2$, form a basis of $\mathbb{R}^{2 \times 2}$ since they are linearly independent ${ }^{2}$. Hence, the criterion of Theorem 4.5 requires to solve the $5 \mathrm{LMI}$ conditions of (9).

\footnotetext{
1 That does not mean that the LMI problem does not admit any solution!

${ }^{2}$ It was checked by a case analysis with Maple 2019.
}

In particular, for $a=8$ and $N=8$, our method provides a positive definite matrix $P$ with 5 LMI conditions instead of 257. Moreover, for these particular choices of $a$ and $N$, the values of the elements $m_{1}, \ldots, m_{4}$ (in Theorem 4.5) are:

$$
m_{1}=m_{2}=m_{3}=m_{4} \sim 423 .
$$

\section{APPENDIX}

In this section, we prove Lemma 4.4. For that, we use the following result, which relates convex hulls to LMI problems.

Leu us recall that given $\mathcal{P}=\left\{\mathbf{p}_{\mathbf{1}}, \cdots, \mathbf{p}_{\mathbf{k}}\right\} \subset \mathbb{R}^{n}$, the convex hull of $\mathcal{P}$ is the set of elements $\sum_{i=1}^{k} a_{i} \mathbf{p}_{\mathbf{i}} \in \mathbb{R}^{n}$, where $a_{i}$ are non-negative real numbers satisfying $\sum_{i=1}^{k} a_{i}=1$.

Lemma 6.1: [26] Let $P=P^{T} \succ 0$ and $M_{i} \in \mathbb{R}^{n \times n}, 1 \leq i \leq$ $k$, such that $P \succ M_{i}^{T} P M_{i}$. If $M \in \mathbb{R}^{n \times n}$ is a matrix in the convex hull of the $M_{i}$ 's, then $P \succ M^{T} P M$.

Given $\lambda \in \mathbb{R}$, we denote by $\operatorname{sign}(\lambda)$ the sign of $\lambda$. Now, we prove Lemma 4.4 .

Proof: [Lemma 4.4] For each non-normal form matrix $A_{\omega_{\ell}}$, recall that $\lambda_{\ell, 1}, \cdots, \lambda_{\ell, d_{N}}$ denote its coordinates in the basis of normal form matrices $A_{\omega_{1}}, \cdots, A_{\omega_{d_{N}}}$. For $1 \leq \ell \leq d_{N}$, (8) is a consequence of the following sequence of inequalities and equality:

$$
P \succ m_{\ell} A_{\omega_{\ell}}^{T} P A_{\omega_{\ell}} \succ \lambda_{\ell, \ell} A_{\omega_{\ell}}^{T} P A_{\omega_{\ell}}=A_{\omega_{\ell}}^{T} P A_{\omega_{\ell}} .
$$

For $d_{N}+1 \leq \ell \leq p^{N}$, we let $n_{\ell}=\sum_{i=1}^{d_{N}}\left|\lambda_{\ell, i}\right|$ and we consider the following decomposition:

$$
\begin{aligned}
A_{\omega_{\ell}} & =\sum_{i=1}^{d_{N}} \lambda_{\ell, i} A_{\omega_{i}} \\
& =\sum_{i=1}^{d_{N}} \frac{\left|\lambda_{\ell, i}\right|}{n_{\ell}}\left(\operatorname{sign}\left(\lambda_{\ell, i}\right) n_{\ell} A_{\omega_{i}}\right) .
\end{aligned}
$$

For $1 \leq i \leq d_{N}$ such that $\lambda_{\ell, i} \neq 0$, by definition of $m_{i}$ in (7) and by definition of $n_{\ell}$, we have $n_{\ell} A_{\omega_{i}} \preceq m_{i} A_{\omega_{i}}$, which implies $P \succ\left(n_{\ell} A_{\omega_{i}}\right)^{T} P\left(n_{\ell} A_{\omega_{i}}\right)=$ $\left(\operatorname{sign}\left(\lambda_{\ell, i}\right) n_{\ell} A_{\omega_{i}}\right)^{T} P\left(\operatorname{sign}\left(\lambda_{\ell, i}\right) n_{\ell} A_{\omega_{i}}\right)$. Hence, (8) follows from Lemma 6.1.

\section{CONCLUSION}

In this paper, we have presented a stability analysis for discrete-time switched linear systems based on linear dependency between matrix words. In particular, we illustrated how this approach may be used to reduced the complexity of LMI systems for checking stability by adding numerical constraints to these LMI. This work admits at least the following two possible extensions. The first one consists in finding better numerical constraints for the LMI problem in order to have a more efficient criterion. The second one is to extend our approach by allowing relations between matrix words of different lengths. In particular, we could use the theory of noncommutative Gröbner [27] bases for constructing bases of the linear span of matrix words. 


\section{REFERENCES}

[1] D. Liberzon, Swithcing in Systems and Control. Systems and Control: Foundation and Applications, Birkhauser, 2003.

[2] R. Shorten, F. Wirth, O. Mason, K. Wulff, and C. King, "Stability criteria for switched and hybrid systems," Invited paper for SIAM Review, vol. 49, no. 4, pp. 545-592, 2007.

[3] R. Alur, A. D'Innocenzo, K. H. Johansson, G. J. Pappas, and G. Weiss, "Compositional modeling and analysis of multi-hop control networks," IEEE Transactions on Automatic Control, vol. 56, pp. 2345-2357, Oct 2011.

[4] L. Hetel, C. Fiter, H. Omran, A. Seuret, E. Fridman, J.-P. Richard, and S. I. Niculescu, "Recent developments on the stability of systems with aperiodic sampling: An overview," Automatica, vol. 76, pp. 309 $-335,2017$.

[5] E. Fridman, Introduction to Time-Delay Systems: Analysis and Control. Systems and Control: Foundations and Applications, Springer International Publishing, 2014.

[6] L. Hetel, J. Daafouz, and C. Iung, "Equivalence between the Lyapunov-Krasovskii functionals approach for discrete delay systems and that of the stability conditions for switched systems," Nonlinear Analysis: Hybrid Systems, vol. 2, no. 3, pp. 697 - 705, 2008. Special Issue Section: Analysis and Design of Hybrid Systems.

[7] V. Blondel and J. N. Tsitsiklis, "The boundedness of all products of a pair of matrices is undecidable," Systems and Control Letters, vol. 41, no. 2, pp. $135-140,2000$.

[8] L. Gurvits, "Stability of discrete linear inclusion," Linear Algebra and its Applications, vol. 231, pp. 47 - 85, 1995.

[9] D. Liberzon, J. Hespanha, and A.-S. Morse, "Stability of switched systems: a Lie-algebraic condition," Systems \& Control Letters, vol. 37, no. 3, pp. 117-122, 1999.

[10] V. Blondel and Y. Nesterov, "Computationally efficient approximations of the joint spectral radius," SIAM Journal on Matrix Analysis and Applications, vol. 27, no. 1, pp. 256-272, 2005.

[11] R. Jungers, The Joint Spectral Radius: Theory and Applications, vol. 385 of Lecture Notes in Control and Information Sciences. Springer-Verlag, 2009.

[12] A. Molchanov and Y. Pyatnitskiy, "Criteria of asymptotic stability of differential and difference inclusions encountered in control theory," Systems \& Control Letters, vol. 13, no. 1, pp. 59-64, 1989.

[13] T. Hu and F. Blanchini, "Non-conservative matrix inequality conditions for stability/stabilizability of linear differential inclusions," Automatica, vol. 46, no. 1, pp. 190-196, 2010.

[14] J. Daafouz, P. Riedinger, and C. Iung, "Stability analysis and control synthesis for switched systems: a switched Lyapunov function approach," IEEE Transactions on Automatic Control, vol. 47, pp. 18831887, Nov 2002.

[15] J.-W. Lee and G. E. Dullerud, "Uniform stabilization of discrete-time switched and Markovian jump linear systems," Automatica, vol. 42, no. 2, pp. $205-218,2006$.

[16] N. Athanasopoulos and M. Lazar, "Alternative stability conditions for switched discrete time linear systems," IFAC Proceedings Volumes, vol. 47, no. 3, pp. 6007-6012, 2014.

[17] A. Megretski, "Integral quadratic constraints derived from the settheoretic analysis of difference inclusions," in 35th IEEE Conference on Decision and Control, vol. 3, pp. 2389 - 2394 vol.3, 011997.

[18] P.-A. Bliman and G. Ferrari-Trecate, "Stability analysis of discretetime switched systems through Lyapunov functions with nonminimal state," IFAC Proceedings Volumes, vol. 36, no. 6, pp. 325 - 329, 2003. IFAC Conference on Analysis and Design of Hybrid Systems 2003, St Malo, Brittany, France, 16-18 June 2003.

[19] A. Kruszewski, R. Wang, and T. M. Guerra, "Nonquadratic stabilization conditions for a class of uncertain nonlinear discrete time ts fuzzy models: A new approach," IEEE Transactions on Automatic Control, vol. 53, pp. 606-611, March 2008.

[20] A. Ahmadi and P. Parrilo, "Non-monotonic Lyapunov functions for stability of discrete time nonlinear and switched systems," in 47th IEEE Conference on Decision and Control, (Cancun, Mexico), pp. 614-621, 2008.
[21] T. S. Gomide and M. J. Lacerda, "Stability analysis of discrete-time switched systems under arbitrary switching," IFAC-PapersOnLine, vol. 51, no. 25, pp. 371 - 376, 2018. 9th IFAC Symposium on Robust Control Design ROCOND 2018.

[22] L. Hetel, A. Kruszewski, W. Perruquetti, and J. P. Richard, "Discretetime switched systems, set-theoretic analysis and quasi-quadratic Lyapunov functions," in 2011 19th Mediterranean Conference on Control Automation (MED), pp. 1325-1330, June 2011.

[23] A. A. Ahmadi and P. A. Parrilo, "Converse results on existence of sum of squares lyapunov functions," in 2011 50th IEEE conference on decision and control and European control conference, pp. 65166521, IEEE, 2011.

[24] A. Aleksandrov, Y. Chen, A. Platonov, and L. Zhang, "Stability analysis for a class of switched nonlinear systems," Automatica, vol. 47, no. 10, pp. 2286 - 2291, 2011.

[25] W. Dayawansa and C. Martin, "A converse Lyapunov theorem for a class of dynamical systems which undergo switching," IEEE Transactions on Automatic Control, vol. 44, no. 4, 1999.

[26] M. C. De Oliveira, J. Bernussou, and J. C. Geromel, "A new discretetime robust stability condition," Systems \& control letters, vol. 37, no. 4, pp. 261-265, 1999.

[27] T. Mora, "An introduction to commutative and noncommutative Gröbner bases," Theoret. Comput. Sci., vol. 134, no. 1, pp. 131-173, 1994. Second International Colloquium on Words, Languages and Combinatorics (Kyoto, 1992). 